\title{
Identifying dynamical systems with bifurcations from noisy partial observation
}

\author{
Yohei Kondo* Kunihiko Kaneko, and Shuji Ishihara \\ Graduate School of Arts and Sciences, University of Tokyo, \\ 3-8-1 Komaba,, Meguro-ku, Tokyo 153-8902, Japan
}

(Dated: June 21, 2021)

\begin{abstract}
Dynamical systems are used to model a variety of phenomena in which the bifurcation structure is a fundamental characteristic. Here we propose a statistical machine-learning approach to derive lowdimensional models that automatically integrate information in noisy time-series data from partial observations. The method is tested using artificial data generated from two cell-cycle control system models that exhibit different bifurcations, and the learned systems are shown to robustly inherit the bifurcation structure.

PACS numbers: $05.45 . T p, 05.45 .-\mathrm{a}, 87.17 . \mathrm{Aa}$
\end{abstract}

Various phenomena ranging from climate change to chemical reactions have been modeled extensively by dynamical systems [1, 2], and the relevance of dynamical systems to modeling biological phenomena is being increasingly recognized [3, 4]. Recent advances in experimental techniques such as live-cell imaging that clarifies molecular activities at high spatiotemporal resolutions [5-7] have accompanied this recognition. However, noise, partial observation, and a low controllability are still challenges for measuring biological systems in that both the system dynamics and measurement processes are highly stochastic, only a few components in a system are observable, and only a small number of experimental conditions can be examined. These difficulties have led to models being constructed from experimental observations in a way that is often ad-hoc and semi-quantitative at best because instructive criteria and practical methods have not yet been established for deriving the model equations by systematically integrating the information in the experimental data.

To model complex systems such as cellular processes, a full description of all the systems details is often impractical and not informative. Instead, a reduced description that preserves the essential features of the system is more useful for comprehension., i.e., models described by low-dimensional dynamical systems are sufficient for explaining experimental observations. In particular, the bifurcation structure is a fundamental feature of dynamical systems since it characterizes the qualitative changes of the dynamics. Thus, identification of low-dimensional model systems that inherit the original bifurcation structure is a crucial step in understanding the dynamics.

Here we propose a statistical machine-learning approach to automatically derive the low-dimensional model equations from single-cell time-series data obtained at a few conditions (i.e., bifurcation parameter values; Fig. 11). Techniques for learning nonlinear dynamical systems from time-series data have been employed for chaos 8, 9], spatiotemporal patterns [10-12], and multi-stable systems [13]. Only a few studies have applied the technique to biological data [14, 15]. In a similar manner to some of those studies [14, 15], we employ a statistical technique to deal with the noisy and partial time-series data. However, rather than aiming to fit the model parameters to the observation, we obtain the low-dimensional model equations that inherit bifurcation structure of the full system to capture the basic nature of the observed system. The performance of the method is demonstrated by using artificial data.

We introduce a nonlinear state space model composed of state and observation equations that describe the system dynamics and observation process, respectively. We consider a system that is modeled by a $D$-dimensional stochastic differential equations, and $d$ components in the model can be simultaneously observed. The state equations are discretized in time by the Euler-Maruyama scheme [16]. We write the time evolution of the $i$ th variable at a time point $t, x_{i}^{t}(i=1, \ldots, D)$, as

$$
x_{i}^{t+1}=x_{i}^{t}+\Delta t f_{i}\left(\left\{x_{j}^{t}\right\}, s\right)+\sigma_{i} \xi_{i}^{t} \sqrt{\Delta t},
$$

where $\Delta t$ is an integration time, $\sigma_{i}$ is the intensity of the system noise, and $s$ is a bifurcation parameter. System noise $\xi_{i}^{t}$ is sampled from a standard normal distribution. To achieve efficient learning, the function $f_{i}$ is considered

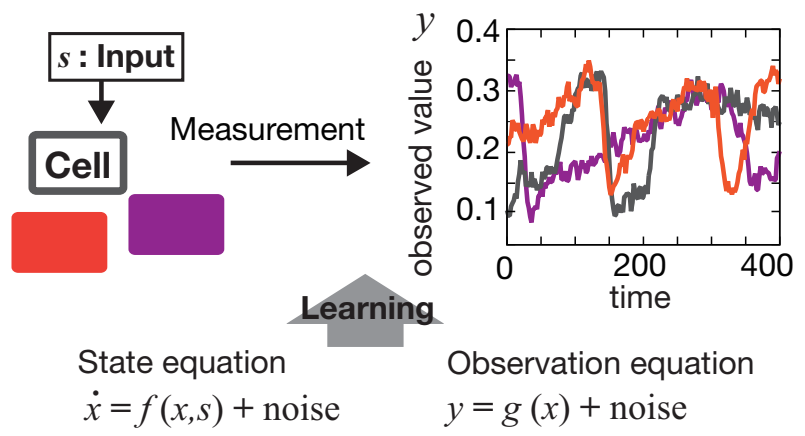

FIG. 1. Schematic representation of the proposed method. Time-series data of the molecular activities in individual cells are obtained from measurements under a given input level $s$, which is regarded as a bifurcation parameter of the system. System and observation equations are then trained to reproduce the time-series data. 
to be expressed by a summation of linearly independent functions as $f_{i}\left(\left\{x_{j}^{t}\right\}, s\right)=\sum_{n}^{N_{i}} k_{i}^{n} f_{i}^{n}\left(\left\{x_{j}\right\}, s\right)$, where $N_{i}$ is the number of parameters $\left\{k_{i}^{n}\right\}$ and functions $\left\{f_{i}^{n}\right\}$. Since our aim is to reproduce the bifurcation structures of systems subjected to unknown equations, we adopt a polynomial base for the $\left\{f_{i}^{n}\right\}$, rather than biochemically realistic functions like the Michaelis-Menten equation.

The observation value of the $i$ th component at a time point $r, y_{i}^{r}(i=1, \ldots, d)$, is written as

$$
y_{i}^{r}=g_{i}\left(x_{i}^{r}\right)+\eta_{i} \phi_{i}^{r}
$$

where $\eta_{i}$ is an observation noise intensity, and $\phi_{i}^{r}$ is sampled from a standard normal distribution. In general, a set of observed time points is a part of the entire set of time points in the numerical integration. Hereafter, $\theta$ indicates the parameters to be estimated: $\left(\left\{k_{i}^{n}\right\},\left\{\sigma_{i}\right\},\left\{\eta_{i}\right\}\right)$.

The learning of dynamical systems is formulated as a maximum likelihood (ML) estimation, which is summarized below (further details are given in the section 1 in the Supplemental Material). The likelihood is given by the conditional probability of the observed time series $Y$ as a function of the model parameters $\theta$. However, a straightforward maximization of the likelihood $p(Y \mid \theta)$ is difficult because it requires the untractable summation of $p(Y \mid X, \theta) p(X \mid \theta)$ with respect to the time series of the state variables $X$. Thus, we employ the EM algorithm to maximize the log likelihood of a model by a two-step iterative method that alternately estimates the states and parameters [17]. In the first step, the E step, the posterior distribution of the time series of a state $(p(X \mid Y, \theta))$ is estimated based on the tentative parameter set $\theta_{\text {old }}$. In the second step, the $\mathrm{M}$ step, the expectation value of $\log p(X, Y \mid \theta)$ is calculated as

$$
Q\left(\theta, \theta_{\text {old }}\right)=\langle\log p(X, Y \mid \theta)\rangle_{p\left(X \mid Y, \theta_{\text {old }}\right)},
$$

and the parameter estimation is updated as

$$
\theta_{\text {new }}=\underset{\theta}{\operatorname{argmax}} Q\left(\theta, \theta_{\text {old }}\right) \text {. }
$$

In this step, the optimization problem is reduced to linear simultaneous equations and thus can be solved easily. However, the problem in the E step is still analytically unsolvable because the probability distribution of the time series is necessary. This calculation requires a state estimation at all time points including the points at which measurements are not conducted. We therefore obtain a numerical approximation of $p(X \mid Y, \theta)$ using a particle filter algorithm that performs state estimations of nonlinear models using a Monte-Carlo method [18, 19]. The particle filter (a numerical extension of Kalman filter) approximates a general non-Gaussian state distribution as a set of particles representing samples from the distribution and evaluates the log likelihood of the models. Since the use of the particle filter introduces stochasticity into the learning algorithm, a slight modification of the $\mathrm{M}$ step is required to ensure convergence of the learning [20]. The optimization function in eq.(4) is replaced by $Q_{I}^{\prime}(\theta)=\left(1-\alpha_{I}\right) Q_{I-1}^{\prime}(\theta)+\alpha_{I} Q\left(\theta, \theta_{\text {old }}\right)$, where $I$ is the iteration index, and $\left\{\alpha_{I}\right\}$ is a sequence of non-increasing positive numbers converging to zero.

To validate the method, we apply it to artificial data generated from models of a eukaryotic cell cycle control system since this system provides an illustrative example of cellular dynamics composed of many molecular components 21 25]. The cell cycle is a fundamental biological process characterized by repeated events underlying cell division and growth in which key proteins, Cyclin and Cyclin-dependent kinases, change their concentration periodically and activate various cellular functions such as DNA synthesis.

Two molecular circuit models of the cell-cycle control system in Xenopus embryos are adopted as the data generators: that proposed by Tyson and co-workers (the Tyson model) 21, 22], and that proposed by Ferrell and co-workers (the Ferrell model) 23, 24]. Although both models show an oscillation onset as the synthesis rate of Cyclin increases, they differ in the type of bifurcation at the onset; the Tyson model exhibits a saddle-node bifurcation on an invariant circle (SNIC), while the Ferrell model exhibits a supercritical Hopf bifurcation. We investigate whether the proposed learning procedure reproduces the correct bifurcation types of each model.

Both data generators are composed of 9 variables including Cdc2, Cyclin, and other regulatory proteins. The time-series data is generated by a numerical calculation of these models as nonlinear Langevin equations at a few parameter values (see the section 2 in the Supplemental Material for the model equations, and obtained time-series data). We simulate noisy observation by adding Gaussian noise to each observation value. Artificial data are prepared for three Cyclin synthesis rates across the bifurcation point, and for each bifurcation parameter value, three independent time series are prepared in which the oscillation exhibits a large fluctuation in amplitude and period among the samples.

Considering a polynomial of degree $M$, we write the system equations to be learned as

$$
f_{i}\left(\left\{x_{j}^{t}\right\}, s\right)=k_{i}^{1}+k_{i}^{2} x_{1}^{t}+k_{i}^{3} x_{2}^{t}+\cdots+k_{i}^{N_{i}}\left(x_{D}^{t}\right)^{M}
$$

The observation equations are expressed simply as $y_{i}^{r}=$ $x_{i}^{r}+\eta_{i} \phi_{i}^{r}$. We consider the active Cdc2 and Cyclin concentrations to be observable variables since their levels have been observed in previous experiments 23]. Accordingly, $y_{1}\left(x_{1}\right)$ and $y_{2}\left(x_{2}\right)$ represent the observed (true) concentrations of active Cdc2 and Cyclin, respectively. The other variables $x_{i}(i>2)$ represent the true concentrations of unobservable components. In the system, the Cyclin synthesis rate, $s$, is a bifurcation parameter. We take the constant term in the equation for Cyclin to be the synthesis rate, i.e., $k_{2}^{1}=s$. Note that the observed orbit in the active Cdc2-Cyclin plane exhibits no 
(a) Tyson model
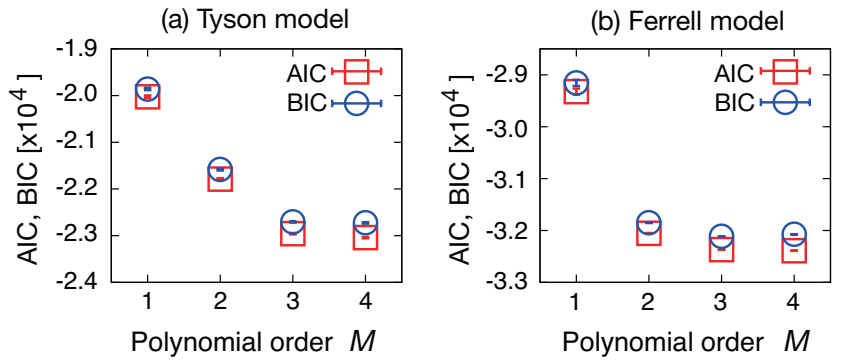

FIG. 2. The AIC and BIC are plotted against different polynomial orders $M=1,2,3,4$ for the (a) Tyson and (b) Ferrell models. For each evaluation, 20 different initial parameters are sampled to avoid the local minima. After the learning, the average and standard deviation are calculated from 100 evaluation trials based on the particle filter.

intersection, (see Fig. S1 in the Supplemental Material) suggesting that the two variables are sufficient to abstract the original high-dimensional dynamics.

The simplest polynomial form required for reproducing the observed dynamics is determined by starting with linear equations composed of active Cdc2 and Cyclin (system dimension $D=2$ and polynomial order $M=1$ ) and increasing the $D$ and $M$ by one. It turns out that $D=2$ is sufficient for reproducing a given time-series data set as shown below. The polynomial order $M$ is determined by minimizing the information criteria through an optimization of the balance between the goodness of fit and the model complexity [26, 27]. The Akaike information criterion (AIC) and Bayesian information criterion (BIC) are evaluated from the log likelihood, parameter number, and data size for each model (Fig,2). Both the AIC and BIC show a decrease from $M=1$ to 3 , but an increase or insignificant decrease at $M=4$. Therefore, we analyze models with $D=2$ and $M=3$ (see the section 3 in the Supplemental Material for the learned parameter values and detailed settings in the learning algorithm).

To check whether the learning procedure can capture the bifurcation of the original data generator system, we compare the bifurcation diagrams of the learned systems with those of the data generators. Figures 3(a) and (b) show bifurcation diagrams against Cyclin synthesis rate $s$ (red lines) for the learned systems in the Tyson and Ferrell models, respectively. The bifurcation diagrams for the corresponding noiseless data generators are shown by the gray lines. Although the data for the learning are given only at three bifurcation parameter points (indicated by the broken lines), the learned systems have quantitatively similar diagrams to those of the corresponding data generators. The sudden appearance of a limit cycle with finite amplitude is reproduced for the Tyson model, while the gradual increase in amplitude from the bifurcation point is reproduced for the Ferrell model. These features are characteristics of the
SNIC and supercritical Hopf bifurcation. Nullclines of the learned systems in the vicinity of the bifurcation points are shown in Figs. 3(c) and (d) for the Tyson model and in Figs. 3(e) and (f) for the Ferrell model. The results confirm the onset of SNIC and supercritical Hopf bifurcation, respectively. Thus, each learned system inherits the bifurcation type of the original model through the learning procedure in spite of noisy and partial observations.

When the learning is conducted by using the data on two of the three bifurcation parameter points, the learned systems still exhibit the correct bifurcation types, although the points of oscillation onset and amplitudes are biased (Figs. 3(g) and (h)). Note that identification of bifurcation is possible even by using the data only on one side of a bifurcation point (as indicated by the green lines). These results indicate the interesting possibility that the learning procedure can predict the type of bifurcation that will occur from the data before the bifurcation point only.

We also show here how the high-dimensional phase space structures of the original data generators are mapped onto the lower-dimensional surfaces in the learned systems. Reduced two-variable models are derived by adiabatic elimination following a similar procedure by Novak and Tyson [28] (see the section 4 in the Supplemental Material for the detailed procedure and reduced model equations). Like the learned systems, the reduced models are composed of active $\mathrm{Cdc} 2$ and total Cyclin. Figure 4 shows the nullclines of the learned systems (the solid orange and purple lines) and the reduced models (the broken lines). In both the Tyson and Ferrell models, the learned system and reduced model nullclines for active Cdc2 have a similar $N$-shaped form (orange lines), indicating the existence of positive feedback in the molecular circuits. In contrast, those for the total Cyclin disagree quite significantly. To check the consistency of the nullclines and dynamics, Fig. 4 also shows a noisy time series from the data generators (blue points) and the orbit of the learned system (red lines). The nullclines of the learned systems are consistent with the dynamics in the data but the reduced models are not. This failure arises because the dynamics of a component mediating the inhibition from active $\mathrm{Cdc} 2$ to Cyclin is not fast enough to allow the adiabatic approximation. Higher-order contribution beyond the adiabatic elimination performed here should be included, which requires complicated technical work. Nevertheless, the learning process automatically reproduces the appropriate low-dimensional dynamics and estimates the bifurcation structures without knowledge of the detailed high-dimensional model systems.

Gathering biological data is complicated by intrinsic and observation noises, partial observation, and a small number of possible experimental conditions. We have outlined here a machine-learning procedure based 

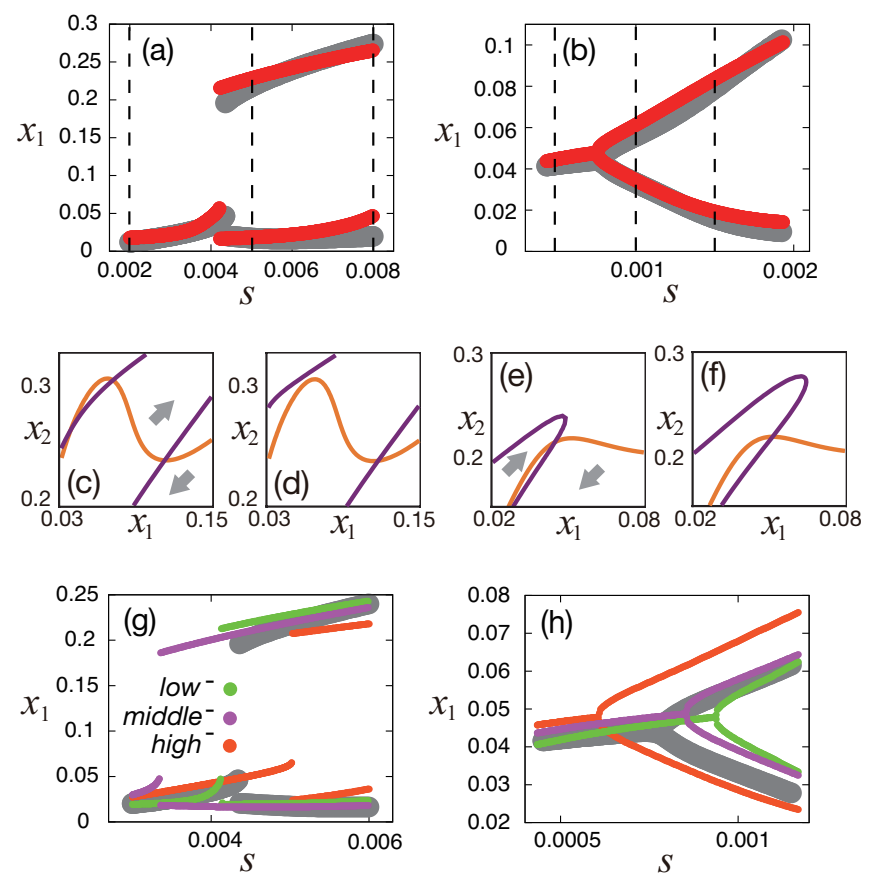

FIG. 3. Bifurcation diagrams of the (a) Tyson and (b) Ferrell models. The active Cdc2 concentration $x_{1}$ for the learned systems is plotted against the Cyclin synthesis rate $s$ (red), and corresponding concentrations of the data generators are also shown for comparison (gray). The broken lines indicate points at which the data are given. For the Tyson model, there is another attractor with a tiny basin that is ignored. (c-f) The nullclines of the learned systems around the bifurcation points are shown. Purple and orange lines represent nullclines of $x_{1}$ (active Cdc2) and $x_{2}$ (Cyclin), respectively, and the gray arrows indicate the flow direction. (c,d) The learned system from the Tyson model exhibits SNIC and $(e, f)$ that from the Ferrell model exhibits a supercritical Hopf bifurcation. The values of the bifurcation parameter are (c) $s=0.0038$, (d) 0.0044 , (e) 0.0005, and (f) 0.0012, respectively. (g,h) Bifurcation diagrams using the data at two of the three bifurcation parameter points. The learning that lacks data at the lowest, intermediate, and highest bifurcation parameter values are denoted by as low ${ }^{-}$, middle $e^{-}$and high $^{-}$, respectively.

on likelihood maximization that makes use of all the information in the time-series data, including that in the noise. By using synthetic data that share the difficulties found in actual biological data, we demonstrated that the procedure could derive low-dimensional model equations that reproduced the obtained time-series data and captured the bifurcation types of the original systems. These results support the conjecture that the learning procedure will be able to construct reliable low-dimensional models for real time-series data of active $\mathrm{Cdc} 2$ and $\mathrm{Cy}-$ clin levels in future studies. Being able to identify the model systems and bifurcation types will provide a useful method for elucidating both the molecular interactions in the circuit and the biological functions of the dynamics.
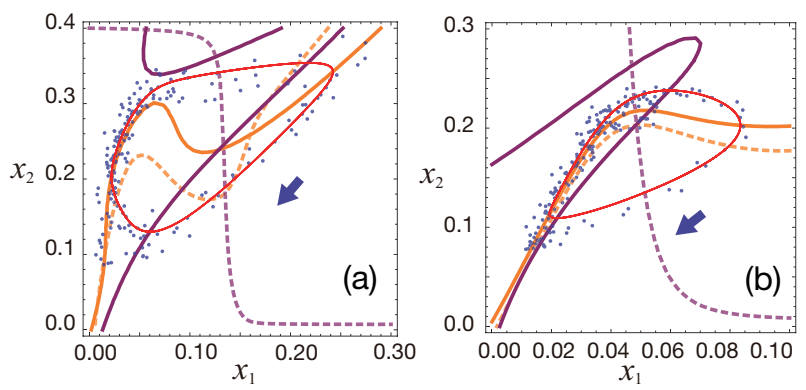

FIG. 4. Comparison of the learned systems and reduced models for the (a) Tyson and (b) Ferrell models. The purple and orange lines represent the nullclines of $x_{1}$ (active $\mathrm{Cdc} 2$ ) and $x_{2}$ (Cyclin), respectively, for the learned systems (solid lines) and reduced models (broken lines). A noisy time series from the data generators (blue dots) and the orbits of the learned models (red lines) are also shown. The blue arrows indicate the flow direction. The values of the bifurcation parameter are (a) $s=0.006$, (b) 0.0015 .

Further, since the dynamics and bifurcation are found widely among various biological processes, the method is expected to be applicable to various cell system with cell-imaging data.

We note that the proposed procedure can be interpreted as a reduction method from high- to lowdimensional systems like adiabatic approximation. In particular, in the vicinity of the bifurcation points, the systems are usually reduced to normal forms represented by low-dimensional differential equations with low-order polynomial forms [29]. However, unlike analytical reduction methods that require the original high-dimensional equations, the present learning procedure uses only the time-series data. This is especially advantageous for studying cell dynamics that involve complex molecular interactions. On the other hand, since the learning method has a less theoretical basis for interpreting the obtained equations, it should be complemented by an analytical procedure.

In essence, the proposed method performs quantitative inference of the phase space structures of the dynamical systems. Therefore, not only the bifurcation structure but also other properties of the dynamical system can be analyzed using the same theoretical groundwork developed here. The detection of phase sensitivity from noisy data in studies of biological clocks [30] would provide an interesting application. In addition, the method is flexible enough to be combined with other machine-learning techniques; it was recently shown that compressive sensing exhibits a high performance for learning dynamical systems [9]. Those possible extensions will further improve our method depending on the situations and experimental setup. In summary, the proposed method will be an efficient way to capture the essential features of the cellular dynamics by mediating dynamical system mod- 
eling with experimental observations.

We would like to thank K. Kamino, N. Saito, and S. Sawai for illuminating comments and stimulating discussions. This work was supported by the Grant-in-Aid MEXT/JSPS (No. 24115503).

* kondo@complex.c.u-tokyo.ac.jp

[1] B. Saltzman, Dynamical Paleoclimatology, Volume 80: Generalized Theory of Global Climate Change (Academic Press, 2001).

[2] S. H. Strogatz, Nonlinear Dynamics And Chaos: With Applications To Physics, Biology, Chemistry, And Engineering (Westview Press, 2001).

[3] K. Kaneko, Life: An Introduction to Complex Systems Biology (Springer, 2006) .

[4] U. Alon, An Introduction to Systems Biology: Design Principles of Biological Circuits (Chapman and Hall/CRC, 2006).

[5] D. J. Stephens and V. J. Allan, Science 300, 82 (2003).

[6] Y. Shav-Tal, R. H. Singer, and X. Darzacq, Nature Rev. Mol. Cell Biol. 5, 855 (2004).

[7] M. Fernández-Suárez and A. Y. Ting, Nature Rev. Mol. Cell Biol. 9, 929 (2008).

[8] K. Judd and A. Mees, Physica D 92, 221 (1996).

[9] W.-X. Wang, R. Yang, Y.-C. Lai, V. Kovanis, and C. Grebogi, Phys. Rev. Lett. 106, 154101 (2011).

[10] H.U. Voss, P. Kolodner, M. Abel, and J. Kurths, Phys. Rev. Lett. 83, 3422 (1999).

[11] T. Müller and J. Timmer, Physica D 171, 1 (2002).

[12] A. Sitz, J. Kurths, and H.U. Voss, Phys. Rev. E 68, 016202 (2003).
[13] J. Ohkubo, Phys. Rev. E 84, 066702 (2011).

[14] M. Nagasaki, R. Yamaguchi, R. Yoshida, S. Imoto, A. Doi, Y. Tamada, H. Matsuno, S. Miyano, and T. Higuchi, Genome inform. 17, 46 (2006).

[15] R. Yoshida, M. Nagasaki, R. Yamaguchi, S. Imoto, S. Miyano, and T. Higuchi, Bioinformatics 24, 2592 (2008).

[16] P. E. Kloeden and E. Platen, Numerical Solution of Stochastic Differential Equations, 3rd ed. (Springer, 1999).

[17] A. P. Dempster, N. M. Laird, and D. B. Rubin, J. R. Statist. Soc. B 39, 1 (1977).

[18] N. Gordon, D. Salmond, and A. Smith, IEE Proceedings-F 140, 107 (1993).

[19] G. Kitagawa, J. Computational and Graphical Statistics 5, 1 (1996).

[20] B. Delyon, M. Lavielle, and E. Moulines, Ann. Statist. 27, 94 (1999).

[21] B. Novak and J. J. Tyson, J. Cell Sci. 106, 1153 (1993).

[22] M.T. Borisuk and J. J. Tyson, J. Theor. Biol. 195, 69 (1998).

[23] J. R. Pomerening, S. Y. Kim, and J. E. Ferrell Jr., Cell 122, 565 (2005).

[24] T. Y.-C. Tsai, Y. S. Choi, W. Ma, J. R. Pomerening, C. Tang, and J. E. Ferrell Jr., Science 321, 126 (2008).

[25] B. Pfeuty, T. David-Pfeuty, and K. Kaneko, Cell Cycle 7, 3246 (2008).

[26] H. Akaike, IEEE Trans. Autom. Control 19, 716 (1974).

[27] G. Schwarz, Ann. Statist. 6, 461 (1978).

[28] B. Novak and J. J. Tyson, J. Theor. Biol. 165, 101 (1993).

[29] J. Guckenheimer and P. Holmes, Nonlinear Oscillations, Dynamical Systems, and Bifurcations of Vector Fields (Springer, 1983).

[30] A. T. Winfree, The Geometry of Biological Time , 2nd ed. (Springer, 2001). 


\title{
Supplemental Material: Identifying dynamical systems with bifurcations from noisy partial observation
}

\author{
Yohei Kondo*, Kunihiko Kaneko, Shuji Ishihara \\ Graduate School of Arts and Sciences, University of Tokyo, 3-8-1 Komaba, Meguro-ku, Tokyo \\ 153-8902, Japan \\ * kondo@complex.c.u-tokyo.ac.jp
}

\section{$1 \quad$ Learning algorithm}

\subsection{State space model}

We first introduce the state space model composed of the state equations and observation equations describing the system dynamics and observation process, respectively. Let us consider $D$-dimensional stochastic differential equations that describe a system, and $d$ components in the system that are observed simultaneously. In the model, the state variable $x_{i}(i=1, \ldots, D)$ evolves under the function $f_{i}\left(\left\{x_{j}\right\}, s\right)$, where $s$ represents an input to the system, and the observation value $y_{i}$ is obtained through the function $g_{i}\left(\left\{x_{j}\right\}\right)$.

By discretizing the dynamics in time with the Euler-Maruyama scheme [1], we can write the space state model as

$$
\begin{aligned}
x_{i}^{t+1} & =x_{i}^{t}+\Delta t f_{i}\left(\left\{x_{j}^{t}\right\}, s\right)+\sigma_{i} \xi_{i}^{t} \sqrt{\Delta t} \\
y_{i}^{r} & =g_{i}\left(\left\{x_{j}^{r}\right\}\right)+\eta_{i} \phi_{i}^{r},
\end{aligned}
$$

where $t(\in T)$ and $r(\in R)$ are time points, $\Delta t$ is an integration time, and $\sigma_{i}$ and $\eta_{i}$ are the noise intensities in the dynamics and observation, respectively. Both $\xi_{i}^{t}$ and $\phi_{i}^{r}$ are sampled from a standard normal distribution. In general, the set of observed time points $R$ is a subset of the entire time point set $T$ (i.e., $R \subseteq T$ ) for the numerical integration. We assume that the function $f_{i}$ can be expressed by the summation of linearly independent functions $\left(f_{i}^{n}\left(n=1, \ldots, N_{i}\right)\right)$ as

$$
f_{i}\left(\left\{x_{j}^{t}\right\}, s\right)=\sum_{n=1}^{N_{i}} k_{i}^{n} f_{i}^{n}\left(\left\{x_{j}^{t}\right\}, s\right) .
$$

Here, $\left\{k_{i}^{n}\right\}$ are the coefficients to be estimated.

Let us consider that $A$ time-series data sets are given. The learning procedure estimates the parameters $\left\{k_{i}^{n}\right\},\left\{\sigma_{i}\right\},\left\{\eta_{i}\right\}$, and all the true states $\left\{x_{i}^{t}\right\}$ for each time-series set. In our method, the initial condition for the $i$ th component in the $a$ th time-series set is assumed to obey a Gaussian distribution parameterized by the mean $\mu_{i, a}$ and the variance $V_{i, a}$. Distributions at other points are automatically estimated by the particle filter algorithm explained below. Then, the parameters to be estimated are $\theta=\left(\left\{k_{i}^{n}\right\},\left\{\sigma_{i}\right\},\left\{\eta_{i}\right\},\left\{\mu_{i, a}\right\},\left\{V_{i, a}\right\}\right)$.

\subsection{SAEM algorithm}

Our aim is to find model parameters $\theta$ by maximizing log likelihood function

$$
\log L(\theta)=\log p(Y \mid \theta)=\log \int_{X} p(Y \mid X, \theta) p(X \mid \theta) .
$$

Here, $Y\left(=\left\{Y_{a}\right\}, a=1, \ldots, A\right)$ denotes the data sets of the $A$ time series, and $X\left(=\left\{X_{a}\right\}, a=\right.$ $1, \ldots, A)$ denotes the entire time series of estimated states. We employ an EM algorithm that maximizes $\log P(X, Y \mid \theta)$ (the complete-data $\log$ likelihood function), which is equivalent to maximizing the likelihood in eq. (4) [2]. By iterating two steps known as the E and M steps, the states $X$ and parameters $\theta$ are estimated alternately. Since our implement of the E step includes the Monte-Carlo method as described below, stochastic approximation EM (SAEM) algorithm is adopted [3]. The SAEM procedure is described as follows. 
1. Initialize the parameter vector $\theta=\theta_{0}$, and set the iteration number $I$ to zero.

2. (E step) Calculate the posterior distribution of the entire time series of state variable $p(X \mid Y, \theta)$.

3. (M step) Rename $\theta$ as $\theta_{\text {old }}$, and update the parameter vector as

$$
\begin{gathered}
\theta_{\text {new }}=\underset{\theta}{\operatorname{argmax}} Q_{I}(\theta) \\
Q_{I}= \begin{cases}Q\left(\theta, \theta_{\text {old }}\right) & (I=0), \\
\left(1-\alpha_{I}\right) Q_{I-1}(\theta)+\alpha_{I} Q\left(\theta, \theta_{\text {old }}\right) & (I>0),\end{cases}
\end{gathered}
$$

where

$$
Q\left(\theta, \theta_{\text {old }}\right)=\langle\log p(X, Y \mid \theta)\rangle_{p\left(X \mid Y, \theta_{\text {old }}\right)},
$$

and $\left\{\alpha_{I}\right\}$ is a non-increasing sequence of positive values converging to zero.

4. Increment $I$ by one, and iterate steps 2 and 3 until the estimation of the parameter vector converges.

The details of the $\mathrm{E}$ and $\mathrm{M}$ steps are described in the following sections.

\section{$1.3 \quad$ E step}

Since different time-series data are independent stochastic variables, we can write

$$
\log p(X \mid Y, \theta)=\sum_{a} \log p\left(X_{a} \mid Y_{a}, \theta\right) .
$$

Then, each $\log p\left(X_{a} \mid Y_{a}, \theta\right)$ is evaluated by using a particle filter algorithm that approximates the non-Gaussian distribution of the state $x_{i}^{t}$ as a collection of many particles, each of which represents a sample from the distribution [4, 5]. Specifically, the algorithm required here is called a particle smoother. For the ath time series, let $x_{i, a}^{t, p}$ denote the $p$ th particle for representing $x_{i}^{t}$, and let $y_{i, a}^{t}$ denote an observed value at time $t$. The procedure of the particle smoother is described as follows.

\section{Particle smoother}

For $a=1, \ldots, A$

1. For $i=1, \ldots, D$ and $p=1, \ldots, P$, set the initial states as $x_{i, a}^{0, p} \sim N\left(\mu_{i, a}, V_{i, a}\right)$, and normalize the weights as $w_{a}^{p}=1 / P$

2. At each $t=0,1, \ldots$

- For $i=1, \ldots, D$ and $p=1, \ldots, P$, calculate $x_{i, a}^{t+1, p}$ from $x_{i, a}^{t, p}$ by using the state equations.

- If $t=r \in R$, update the weights of the particles as

$$
w_{a}^{p}=\frac{w_{a, \text { old }}^{p} l_{a}^{r, p}}{\sum_{p} w_{a, \text { old }}^{p} l_{a}^{r, p}},
$$

where

$$
l_{a}^{r, p}=\prod_{i}^{d} p\left(y_{i, a}^{r} \mid\left\{x_{j, a}^{r, p}\right\}\right) .
$$


- If $P_{\text {eff }}=1 / \sum_{p}\left(w_{a}^{p}\right)^{2}<P_{\text {thres }}$ (i.e., if effective number of the particles fall below a threshold value), resample the particles according to the new weights. Note that the history of particles $\left(x_{i, a}^{0, p}, x_{i, a}^{1, p}, \ldots, x_{i, a}^{r-1, p}\right)$ is resampled in parallel.

3. Finish when all data points have passed $(t=\max (R))$, and estimate the log likelihood as

$$
\log L_{a}(\theta)=\sum_{r \in R} \log \left(\frac{1}{P} \sum_{p}^{P} l_{a}^{r, p}\right) .
$$

Then, the posterior distribution of the time series of the state is approximated as

$$
p\left(X_{a} \mid Y_{a}, \theta_{\text {old }}\right)=\sum_{p=1}^{P} w_{a}^{p} \delta\left(X_{a}-X_{a}^{p}\right),
$$

where $X_{a}^{p}$ indicates a sample path $\left(\left\{x_{i, a}^{t, p}\right\}, i=1, \ldots, D, t \in T\right)$. On the basis of this approximation, we calculate the average of the complete-data log likelihood as

$$
\begin{aligned}
Q\left(\theta, \theta_{\text {old }}\right) & =\left\langle\log p\left(\left\{X_{a}\right\},\left\{Y_{a}\right\} \mid \theta\right)\right\rangle_{p\left(\left\{X_{a}\right\} \mid\left\{Y_{a}\right\}, \theta_{\text {old }}\right)} \\
& =\sum_{a=1}^{A} \sum_{p=1}^{P} w_{a}^{p} \log p\left(X_{a}^{p}, Y_{a} \mid \theta\right) \\
& =\sum_{a=1}^{A} \sum_{p=1}^{P} \sum_{i=1}^{D} w_{a}^{p}\left(-\frac{1}{2} \log 2 \pi V_{i, a}-\frac{\left(x_{i, a}^{0, p}-\mu_{i, a}\right)^{2}}{2 V_{i, a}}\right) \\
& +\sum_{a=1}^{A} \sum_{p=1}^{P} \sum_{t \in T} \sum_{i=1}^{D} w_{a}^{p}\left(-\frac{1}{2} \log 2 \pi\left(\sigma_{i}\right)^{2}-\frac{\left(\left(x_{i, a}^{t+1, p}-x_{i, a}^{t, p}\right)-\Delta t \sum_{n=1}^{N_{i}} k_{i}^{n} f_{i}^{n}\left(\left\{x_{j, a}^{t, p}\right\}\right)\right)^{2}}{2\left(\sigma_{i}\right)^{2} \Delta t}\right) \\
& +\sum_{a=1}^{A} \sum_{p=1}^{P} \sum_{r \in R} \sum_{i=1}^{d} w_{a}^{p}\left(-\frac{1}{2} \log 2 \pi\left(\eta_{i}\right)^{2}-\frac{\left(y_{i, a}^{r, p}-g_{j}\left(\left\{x_{j, a}^{r, p}\right\}\right)\right)^{2}}{2\left(\eta_{j}\right)^{2}}\right) .
\end{aligned}
$$

\section{$1.4 \quad$ M step}

At the $I$ th iteration, the parameter-value update is performed by finding the $\theta$ for which $\frac{d}{d \theta} Q_{I}(\theta)=0$. We describe the case of $\frac{d}{d \theta} Q\left(\theta, \theta_{\text {old }}\right)=0$ for simplicity, although the optimization problem can be solved generally by the same method. The following example demonstrates the determination of parameters of the system dynamics $\left(k_{i}^{n}\right)$ and the strength of the system noise $\left(\sigma_{i}\right)$ in detail.

First, by differentiating the complete-data log likelihood with respect to $k_{l}^{m}$, we obtain

$$
\begin{aligned}
0 & =\frac{\partial}{\partial k_{l}^{m}} Q\left(\theta, \theta_{\mathrm{old}}\right) \\
& =\sum_{a}^{A} \sum_{p}^{P} \sum_{t \in T} w_{a}^{p}\left(\frac{\left(\left(x_{l, a}^{t+1, p}-x_{l, a}^{t, p}\right)-\Delta t \sum_{n}^{N_{l}} k_{l}^{n} f_{l}^{n}\left(\left\{x_{j, a}^{t, p}\right\}\right)\right) \Delta t f_{l}^{m}\left(\left\{x_{j, a}^{t, p}\right\}\right)}{2\left(\sigma_{l}\right)^{2} \Delta t}\right) \\
& =\sum_{a, p, t} w_{a}^{p}\left(\Delta x_{l, a}^{t, p} f_{l}^{m}\left(\left\{x_{j, a}^{t, p}\right\}\right)\right)-\left(\sum_{n}^{N_{l}} k_{l}^{n} \sum_{a, p, t} w_{a}^{p} f_{l}^{n}\left(\left\{x_{j, a}^{t, p}\right\}\right) f_{l}^{m}\left(\left\{x_{j, a}^{t, p}\right\}\right)\right),
\end{aligned}
$$


where $\Delta x_{l, a}^{t, p}=\left(x_{l, a}^{t+1, p}-x_{l, a}^{t, p}\right) / \Delta t$. By defining the vectors $b_{l}$ and $k_{l}$ and a matrix $A_{l}$ as

$$
\begin{aligned}
\left(b_{l}\right)_{m} & =\sum_{a, p, t} w_{a}^{p}\left(\Delta x_{l, a}^{t, p} f_{l}^{m}\left(\left\{x_{j, a}^{t, p}\right\}\right)\right) \\
\left(k_{l}\right)_{m} & =k_{l}^{m} \\
\left(A_{l}\right)_{n m} & =\sum_{a, p, t} w_{a}^{p} f_{l}^{n}\left(\left\{x_{j, a}^{t, p}\right\}\right) f_{l}^{m}\left(\left\{x_{j, a}^{t, p}\right\}\right),
\end{aligned}
$$

the system dynamics parameters are determined as follows.

$$
k_{l}=\left(A_{l}\right)^{-1} b_{l} .
$$

Next, using the new $k_{i}^{n}$ calculated above, we obtain

$$
\frac{\partial}{\partial \sigma_{l}} Q\left(\theta, \theta_{\text {old }}\right)=\sum_{a, p, t} w_{a}^{p}\left(-\frac{1}{\sigma_{l}}+\frac{\Delta t^{2}\left(\Delta x_{l, a}^{t, p}-\sum_{n}^{N_{l}} k_{l}^{n} f_{l}^{n}\left(\left\{x_{j, a}^{t, p}\right\}\right)\right)}{\left(\sigma_{l}\right)^{3} \Delta t}\right)=0
$$

and thus,

$$
\left(\sigma_{l}\right)^{2}=\Delta t \sum_{a, p, t} w_{a}^{p}\left(\Delta x_{l, a}^{t, p}-\sum_{n}^{N_{l}} k_{l}^{n} f_{l}^{n}\left(\left\{x_{j, a}^{t, p}\right\}\right)\right)^{2} .
$$

The other parameters are estimated in the same manner. Only for the variance of the initial condition $V_{i, a}$, we define the minimum value $V_{\min }$ to avoid an unnaturally small value resulting from a problem called sample impoverishment [6].

\section{Data preparation}

The model equations and parameter values used in the present study as the data generators are described here. Each data generators, the Tyson and Ferrell models, is numerically integrated with white Gaussian noise by using a stochastic Runge-Kutta II (SRKII) algorithm [7]. To prohibit negative values of the chemical concentrations as a result of noise, each variable is reset to a small positive value $\epsilon(=0.001)$ when the value is less than $\epsilon$. We confirmed that the results of the present study are stable so long as $\epsilon$ is sufficiently small.

The Tyson model described in Novak \& Tyson [8].

$$
\begin{aligned}
& \frac{d[\text { cyclin }]}{d t}=k_{1}[\mathrm{AA}]-k_{2}[\mathrm{cyclin}]-k_{3}[\mathrm{Cdc} 2][\operatorname{cyclin}]+\xi_{1}(t) \\
& \frac{d[\mathrm{Cdc} 2 \text {-cyclin }]}{d t}=k_{P P}[\mathrm{Cdc} 2 \text {-cyclin-tp }]-\left(k_{w e e}+k_{c a k}+k_{2}\right)[\mathrm{Cdc} 2 \text {-cyclin }] \\
& +\quad k_{c d c 25}\left[\mathrm{Cdc} 2 \text {-cyclin-yp] }+k_{3}[\mathrm{Cdc2}][\text { cyclin }]+\xi_{2}(t)\right. \\
& \frac{d[\mathrm{Cdc} 2 \text {-cyclin-yp }]}{d t}=k_{w e e}[\mathrm{Cdc} 2 \text {-cyclin }]-\left(k_{c d c 25}+k_{c a k}+k_{2}\right)[\mathrm{Cdc} 2 \text {-cyclin-yp }] \\
& +\quad k_{P P}[\mathrm{Cdc} 2 \text {-cyclin-yptp }]+\xi_{3}(t) \\
& \frac{d[\text { Cdc2-cyclin-yptp }]}{d t}=k_{w e e}[\text { Cdc2-cyclin-tp }]-\left(k_{P P}+k_{c d c 25}+k_{2}\right)[\text { Cdc2-cyclin-yptp] } \\
& +\quad k_{c a k}[\text { Cdc2-cyclin-yp }]+\xi_{4}(t)
\end{aligned}
$$




$$
\begin{aligned}
& \frac{d[\text { Cdc2-cyclin-tp }]}{d t}=k_{\text {cak }}[\text { Cdc2-cyclin }]-\left(k_{P P}+k_{w e e}+k_{2}\right)[\text { Cdc2-cyclin-tp }] \\
& +k_{c d c 25}[\mathrm{Cdc2} 2 \text {-cyclin-yptp }]+\xi_{5}(t) \\
& \frac{d\left[\mathrm{Cdc} 25^{*}\right]}{d t}=k_{a}[\mathrm{Cdc} 2 \text {-cyclin-tp }] \frac{\left(\left[\mathrm{Cdc} 25_{t o t}\right]-\left[\mathrm{Cdc} 25^{*}\right]\right)}{K_{a}+\left(\left[\mathrm{Cdc} 25_{t o t}\right]-\left[\mathrm{Cdc} 25^{*}\right]\right)} \\
& -\quad k_{b}[\mathrm{PPase}] \frac{\left[\mathrm{Cdc} 25^{*}\right]}{K_{b}+\left[\mathrm{Cdc} 25^{*}\right]}+\xi_{6}(t) \\
& \frac{d\left[\mathrm{APC}^{*}\right]}{d t}=k_{c}\left[\mathrm{IEP}^{*}\right] \frac{\left(\left[\mathrm{APC}_{t o t}\right]-\left[\mathrm{APC}^{*}\right]\right)}{K_{c}+\left(\left[\mathrm{APC}_{t o t}\right]-\left[\mathrm{APC}^{*}\right]\right)}-k_{d}[\operatorname{anti-} \mathrm{IE}] \frac{\left[\mathrm{APC}^{*}\right]}{K_{d}+\left[\mathrm{APC}^{*}\right]}+\xi_{7}(t) \\
& \frac{d\left[\mathrm{Wee}^{*}\right]}{d t}=k_{e}[\mathrm{Cdc} 2 \text {-cyclin-tp }] \frac{\left(\left[\mathrm{Wee} 1_{t o t}\right]-\left[\mathrm{Wee}^{*}\right]\right)}{K_{e}+\left(\left[\mathrm{Wee} 1_{t o t}\right]-\left[\mathrm{Wee}^{*}\right]\right)} \\
& -\quad k_{f}[\mathrm{PPase}] \frac{\left[\mathrm{Wee}^{*}\right]}{K_{f}+\left[\mathrm{Wee}^{*}\right]}+\xi_{8}(t) \\
& \frac{d\left[\mathrm{IEP}^{*}\right]}{d t}=k_{g}[\mathrm{Cdc} 2-\mathrm{cyclin}-\mathrm{tp}] \frac{\left(\left[\mathrm{IE}_{t o t}\right]-\left[\mathrm{IEP}^{*}\right]\right)}{K_{g}+\left(\left[\mathrm{IE}_{t o t}\right]-\left[\mathrm{IEP}^{*}\right]\right)} \\
& -\quad k_{h}[\mathrm{PPase}] \frac{\left[\mathrm{IEP}^{*}\right]}{K_{h}+\left[\mathrm{IEP}^{*}\right]}+\xi_{9}(t) \\
& k_{c d c 25}=V_{c d c 25^{\prime}}\left(\left[\mathrm{Cdc} 25_{t o t}\right]-\left[\mathrm{Cdc} 25^{*}\right]\right)+V_{c d c 25^{\prime \prime}}\left[\mathrm{Cdc} 25^{*}\right] \\
& k_{\text {wee }}=V_{\text {wee }^{\prime}}\left[\mathrm{Wee}^{*}\right]+V_{\text {wee }^{\prime \prime}}\left(\left[\mathrm{Wee}_{\text {tot }}\right]-\left[\mathrm{Wee}^{*}\right]\right) \\
& k_{2}=V_{2^{\prime}}\left(\left[\mathrm{APC}_{t o t}\right]-\left[\mathrm{APC}^{*}\right]\right)+V_{2^{\prime \prime}}\left[\mathrm{APC}^{*}\right] \\
& {[\mathrm{Cdc} 2]=\left[\mathrm{Cdc} 2_{\text {tot }}\right]-[\mathrm{Cdc} 2-\text { cyclin }]-[\mathrm{Cdc} 2 \text {-cyclin-yp }]} \\
& \text { - [Cdc2-cyclin-yptp] - [Cdc2-cyclin-tp] }
\end{aligned}
$$

Noise terms are represented by $\xi_{i}(t)$ with white Gaussian statistics $\left\langle\xi_{i}(t)\right\rangle=0$ and $\left\langle\xi_{i}(t) \xi_{j}(\tau)\right\rangle=$ $2 \sigma_{i}^{2} \delta_{i, j} \delta(t-\tau)$.

\section{$2.1 \quad$ Ferrell model}

The Ferrell model is described in Tsai et al. [9].

$$
\begin{aligned}
& \frac{d[\text { cyclin }]}{d t}=k_{\text {synth }}-k_{\text {dest }}\left[\mathrm{APC}^{*}\right][\text { cyclin }]-k_{a}[\mathrm{Cdc} 2][\text { cyclin }]+k_{d}[\mathrm{Cdc} 2-\mathrm{cyclin}]+\xi_{1}(t) \\
& \frac{d[\mathrm{Cdc} 2-\mathrm{cyclin}]}{d t}=k_{a}[\mathrm{Cdc} 2][\mathrm{cyclin}]-k_{d}[\mathrm{Cdc} 2-\mathrm{cyclin}]-k_{\text {dest }}\left[\mathrm{APC}^{*}\right][\mathrm{Cdc} 2-\mathrm{cyclin}] \\
& \text { - } k_{\text {wee1 }}\left[\text { Wee1 }{ }^{*}\right]\left[\text { Cdc2-cyclin] }-k_{\text {wee1basal }}\left(\left[\mathrm{Wee}_{\text {tot }}\right]-\left[\mathrm{Wee}^{*}\right]\right)[\mathrm{Cdc} 2 \text {-cyclin] }\right. \\
& +k_{C d c 25}\left[\mathrm{Cdc} 25^{*}\right][\mathrm{Cdc} 2 \text {-cyclin-yp }]+k_{C d c 25 b a s a l}\left(\left[\mathrm{Cdc} 25_{t o t}\right]-\left[\mathrm{Cdc} 25^{*}\right]\right)[\mathrm{Cdc} 2 \text {-cyclin-y] } \\
& +\xi_{2}(t) \\
& \frac{d[\mathrm{Cdc} 2 \text {-cyclin-yp }]}{d t}=k_{\text {wee } 1}\left[\mathrm{Wee}^{*}\right][\mathrm{Cdc} 2 \text {-cyclin }]+k_{\text {wee1basal }}\left(\left[\mathrm{Wee} 1_{\text {tot }}\right]-\left[\mathrm{Wee}^{*}\right]\right)[\mathrm{Cdc} 2 \text {-cyclin }] \\
& -\quad k_{C d c 25}\left[\mathrm{Cdc} 25^{*}\right][\mathrm{Cdc} 2 \text {-cyclin-yp }]-k_{C d c 25 b a s a l}\left(\left[\mathrm{Cdc} 25_{t o t}\right]-\left[\mathrm{Cdc} 25^{*}\right]\right)[\mathrm{Cdc} 2 \text {-cyclin-yp] } \\
& -\quad k_{c a k}[\mathrm{Cdc} 2 \text {-cyclin-yp }]+k_{p p 2 c}[\mathrm{Cdc} 2 \text {-cyclin-yptp }]-k_{\text {dest }}\left[\mathrm{APC}^{*}\right][\mathrm{Cdc2} \text {-cyclin-yp }] \\
& +\xi_{3}(t) \\
& \frac{d[\mathrm{Cdc} 2 \text {-cyclin-yptp }]}{d t}=k_{c a k}[\mathrm{Cdc2} 2 \text {-cyclin-yp }]-k_{p p 2 c}[\mathrm{Cdc} 2 \text {-cyclin-yptp }] \\
& -k_{C d c 25}\left[\mathrm{Cdc} 25^{*}\right][\mathrm{Cdc} 2-c y c l i n-y p t p]-k_{C d c 25 b a s a l}\left(\left[\mathrm{Cdc} 25_{t o t}\right]-\left[\mathrm{Cdc} 25^{*}\right]\right)[\mathrm{Cdc} 2 \text {-cyclin-yptp] } \\
& +k_{\text {wee1 }}\left[\text { Wee1 }{ }^{*}\right][\text { Cdc2-cyclin-tp }]+k_{\text {wee1basal }}\left(\left[\mathrm{Wee}_{\text {tot }}\right]-\left[\text { Wee1 }^{*}\right]\right)[\text { Cdc2-cyclin-tp] } \\
& -k_{\text {dest }}\left[\mathrm{APC}^{*}\right][\mathrm{Cdc2} \text {-cyclin-yptp }]+\xi_{4}(t)
\end{aligned}
$$




$$
\begin{aligned}
& \frac{d[\mathrm{Cdc} 2 \text {-cyclin-tp }]}{d t}=k_{C d c 25}\left[\mathrm{Cdc} 25^{*}\right][\mathrm{Cdc} 2 \text {-cyclin-yptp }] \\
& +k_{\text {Cdc25basal }}\left(\left[\mathrm{Cdc} 25_{t o t}\right]-\left[\mathrm{Cdc} 25^{*}\right]\right)[\mathrm{Cdc} 2 \text {-cyclin-yptp }] \\
& -k_{\text {wee } 1}\left[\mathrm{Wee}^{*}\right]\left[\mathrm{Cdc} 2 \text {-cyclin-tp] }-k_{\text {wee1basal }}\left(\left[\mathrm{Wee}_{\text {tot }}\right]-\left[\mathrm{Wee1}^{*}\right]\right)[\mathrm{Cdc} 2 \text {-cyclin-tp] }\right. \\
& -k_{\text {dest }}\left[\mathrm{APC}^{*}\right][\mathrm{Cdc} 2 \text {-cyclin-tp }]+\xi_{5}(t) \\
& \frac{d\left[\mathrm{Cdc} 25^{*}\right]}{d t}=k_{C d c 25 o n} \frac{[\mathrm{Cdc} 2-\mathrm{cyclin}-\mathrm{tp}]^{n_{c d c 25}}}{\left.\operatorname{EC} 50_{C d c 25}^{n_{c d c 25}}+[\mathrm{Cdc} 2-\mathrm{cyclin}-\mathrm{tp}]\right]^{n_{c d c 25}}}\left(\left[\mathrm{Cdc} 25_{t o t}\right]-\left[\mathrm{Cdc} 25^{*}\right]\right) \\
& -k_{C d c 25 o f f}\left[\mathrm{Cdc} 25^{*}\right]+\xi_{6}(t)
\end{aligned}
$$

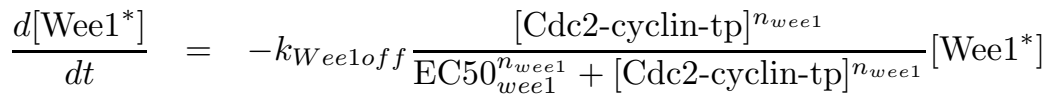

$$
\begin{aligned}
& +k_{W e e 1 o n}\left(\left[\text { Wee }_{\text {tot }}\right]-\left[\text { Wee1 }^{*}\right]\right)+\xi_{7}(t) \\
& \frac{d\left[\mathrm{Plx}^{*}\right]}{d t}=k_{p l x o n} \frac{[\mathrm{Cdc} 2-\mathrm{cyclin}-\mathrm{tp}]^{n_{p l x}}}{\mathrm{EC} 50_{p l x}^{n_{p l x}}+[\mathrm{Cdc} 2-\mathrm{cyclin}-\mathrm{tp}]^{n_{p l x}}}\left(\left[\mathrm{Plx}_{t o t}\right]-\left[\mathrm{Plx}^{*}\right]\right) \\
& -k_{\text {plxoff }}\left[\mathrm{Plx}^{*}\right]+\xi_{8}(t) \\
& \frac{d\left[\mathrm{APC}^{*}\right]}{d t}=k_{a p c o n} \frac{\left[\mathrm{Plx}^{*}\right]^{n_{a p c}}}{\mathrm{EC} 50_{a p c}^{n_{p p c}}+\left[\mathrm{Plx}^{*}\right]^{n_{a p c}}}\left(\left[\mathrm{APC}_{t o t}\right]-\left[\mathrm{APC}^{*}\right]\right)-k_{a p c o f f}\left[\mathrm{APC}^{*}\right]+\xi_{9}(t) \\
& \left.[\mathrm{Cdc} 2]=\left[\mathrm{Cdc} 2_{t o t}\right]-[\mathrm{Cdc} 2-c y c l i n]-[\text { Cdc2-cyclin-yp }]-\text { [Cdc2-cyclin-yptp }\right]-[\text { Cdc2-cyclin-tp }]
\end{aligned}
$$

Noise terms are represented by $\xi_{i}(t)$ with white Gaussian statistics $\left\langle\xi_{i}(t)\right\rangle=0$ and $\left\langle\xi_{i}(t) \xi_{j}(\tau)\right\rangle=$ $2 \sigma_{i}^{2} \delta_{i, j} \delta(t-\tau)$.

\section{$2.2 \quad$ Artificial measurement process}

For both the Tyson and Ferrell models, the artificial measurement process is implemented as follows.

$$
\begin{aligned}
y_{1}= & \text { active } \mathrm{Cdc} 2=\frac{[\text { Cdc2-cyclin-tp }]+\eta_{1} \phi_{1}}{\left[\mathrm{Cdc} 2_{\mathrm{tot}}\right]} \\
y_{2}= & \text { Total Cyclin } \\
= & ([\text { cyclin }]+[\mathrm{Cdc} 2 \text {-cyclin }]+[\mathrm{Cdc} 2 \text {-cyclin-yp }]+[\text { Cdc2-cyclin-yptp }] \\
& \left.+[\mathrm{Cdc} 2 \text {-cyclin-tp }]+\eta_{2} \phi_{2}\right) /\left[\mathrm{Cdc} 2_{\mathrm{tot}}\right] .
\end{aligned}
$$

Here, $\eta_{1,2}$ is the observation noise intensity, and $\phi_{1,2}$ is sampled from a standard normal distribution.

\subsection{Parameter values}

The parameters used in the Tyson and Ferrell models are listed in Tables \$1 and \$2, respectively. In the Tyson model, all the parameter values are the same as those in the original literature [8]. In the Ferrell model, all the parameter values are the same as those in Pomerening et al. [10] except for "factor," which is set as in [9].

\subsection{Orbits of the data generators}

Figure \$1 show noiseless orbits of the data generators. We note that each orbit exhibits no intersection, indicating that the two observable variables are sufficient to abstract the original high-dimensional dynamics.

\subsection{Data set}

The data used for the learning are shown in supplemental Fig. \$2, 


\section{Settings and results of learning}

Parameters in the learning algorithm is listed in Table $\mathrm{S3}$. The learned parameters of the third-order polynomial model used in the main text is shown in Table $\mathbf{\$ 4}$.

\section{Model reduction}

We reduce the Tyson and Ferrell models to two-dimensional systems by the same procedure as described in [11]. By denoting the non-dimensionalized active Cdc2 and total Cyclin levels as $u$ and $v$, respectively, the reduced models are written as follows.

The reduced Tyson model takes the form as

$$
\begin{aligned}
\dot{u} & =\frac{s}{1+k_{P P} / k_{c a k}}-\left(f_{\mathrm{APC}}(u)+f_{\mathrm{Wee}}(u)\right) u+f_{\mathrm{Cdc} 25}(u)\left(\frac{v}{1+k_{P P} / k_{c a k}}-u\right), \\
\dot{v} & =s-f_{\mathrm{APC}}(u) v
\end{aligned}
$$

where $f_{\mathrm{Cdc} 25}, f_{\mathrm{Wee}}$, and $f_{\mathrm{APC}}$ are the functions corresponding to the adiabatic solutions of eqs. (32), (33), and (34), respectively.

This reduction procedure includes the determination of the level of Cdc2-Cyclin-tp (i.e., the value of $u$ ) from the sum [Cdc2-Cyclin] + [Cdc2-Cyclin-tp] (see Appendix A in [11]). This is based on a detailed balance assumption of the phosphorylation reaction between the two molecular species. However, in the Ferrell model, the absence of the reaction makes the original reduction procedure inapplicable. Then, we simply assume [Cdc2-Cyclin-tp] $[$ Cdc2-Cyclin] + [Cdc2-Cyclin-tp], because it is observed that the ratio of [Cdc2-Cyclin] to the summation is small throughout the dynamics within the parameter region we consider. Consequently, the reduced Ferrell model is written as

$$
\begin{aligned}
& \left.\dot{u}=s-\left(f_{\mathrm{APC}}(u)\right)+f_{\mathrm{Wee}}(u)\right) u+f_{\mathrm{Cdc} 25}(v-u), \\
& \dot{v}=s-f_{\mathrm{APC}}(u) v
\end{aligned}
$$

where $f_{\mathrm{Cdc} 25}, f_{\mathrm{Wee}}$, and $f_{\mathrm{APC}}$ are derived from the adiabatic approximation in the same manner as the Tyson model. 


\begin{tabular}{|c|c|}
\hline Parameter & \\
\hline 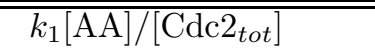 & $\overline{s s}$ \\
\hline$k_{3}\left[\mathrm{Cdc} 2_{t o t}\right]$ & 1.0 \\
\hline$k_{C A K}$ & 0.25 \\
\hline$k_{P P}$ & 0.025 \\
\hline$V_{2^{\prime}}\left[\mathrm{APC}_{t o t}\right]$ & 0.015 \\
\hline$V_{2^{\prime \prime}}\left[\mathrm{APC}_{t o t}\right]$ & 1.0 \\
\hline$V_{c d c 25^{\prime}}\left[\mathrm{Cdc} 25_{t o t}\right]$ & 0.1 \\
\hline$V_{c d c 25^{\prime \prime}}\left[\mathrm{Cdc} 25_{t o t}\right]$ & 2.0 \\
\hline$V_{w e e^{\prime}}\left[\mathrm{Wee} 1_{t o t}\right]$ & 0.1 \\
\hline$V_{w e e^{\prime \prime}}\left[\mathrm{Wee} 1_{t o t}\right]$ & 1.0 \\
\hline$k_{a}\left[\mathrm{Cdc} 2_{t o t}\right] /\left[\mathrm{Cdc} 25_{t o t}\right]$ & 1.0 \\
\hline$k_{b}[\mathrm{PPase}] /\left[\mathrm{Cdc} 25_{t o t}\right]$ & 0.125 \\
\hline$k_{c}\left[\mathrm{IE}_{t o t}\right] /\left[\mathrm{APC}_{t o t}\right]$ & 0.1 \\
\hline$k_{d}[$ anti IE $] /\left[\mathrm{APC}_{t o t}\right]$ & 0.095 \\
\hline$k_{e}\left[\mathrm{Cdc} 2_{t o t}\right] /\left[\mathrm{Wee} 1_{t o t}\right]$ & 1.33 \\
\hline$k_{f}[\mathrm{PPase}] /\left[\mathrm{Wee} 1_{t o t}\right]$ & 0.1 \\
\hline$k_{q}\left[\mathrm{Cdc} 2_{t o t}\right] /\left[\mathrm{IE}_{t o t}\right]$ & 0.65 \\
\hline$k_{h}[\mathrm{PPase}] /\left[\mathrm{IE}_{t o t}\right]$ & 0.087 \\
\hline$K_{a} /\left[\mathrm{Cdc} 25_{t o t}\right]$ & 0.1 \\
\hline$K_{b} /\left[\mathrm{Cdc} 25_{t o t}\right]$ & 0.1 \\
\hline$K_{c} /\left[\mathrm{APC}_{t o t}\right]$ & 0.01 \\
\hline$K_{d} /\left[\mathrm{APC}_{t o t}\right]$ & 0.01 \\
\hline$K_{e} /\left[\right.$ Wee $\left.1_{t o t}\right]$ & 0.3 \\
\hline$K_{f} /\left[\right.$ Wee $\left.1_{t o t}\right]$ & 0.3 \\
\hline$K_{g} /\left[\mathrm{IE}_{t o t}\right]$ & 0.01 \\
\hline$K_{h} /\left[\mathrm{IE}_{t o t}\right]$ & 0.01 \\
\hline$\sigma_{1, \ldots, 5} /\left[\mathrm{Cdc} 2_{t o t}\right]$ & 0.0024 \\
\hline$\sigma_{6} /\left[\mathrm{Cdc} 25_{t o t}\right]$ & 0.0024 \\
\hline$\sigma_{7} /\left[\mathrm{APC}_{t o t}\right]$ & 0.0024 \\
\hline$\sigma_{8} /\left[\mathrm{Wee}_{t o t}\right]$ & 0.0024 \\
\hline$\sigma_{9} /\left[\mathrm{IEP}_{t o t}\right]$ & 0.0024 \\
\hline$\eta_{1} /\left[\mathrm{Cdc} 2_{t o t}\right]$ & 0.005 \\
\hline$\eta_{2} /\left[\mathrm{Cdc} 2_{t o t}\right]$ & 0.005 \\
\hline
\end{tabular}

Table S1: Parameters in the Tyson model

\begin{tabular}{|c|c|}
\hline Parameter & \\
\hline$k_{\text {syn }}$ & $s$ \\
\hline$k_{\text {dest }}$ & 0.006 \\
\hline$k_{a}$ & 0.1 \\
\hline$k_{d}$ & 0.001 \\
\hline factor & 10 \\
\hline$k_{w e e 1}$ & 0.05 \\
\hline$k_{\text {wee1basal }}$ & $k_{w e e 1} /$ factor \\
\hline$k_{C d c 25}$ & 0.1 \\
\hline$k_{C d c 25 b a s a l}$ & $k_{c d c 25} /$ factor \\
\hline$k_{c a k}$ & 0.8 \\
\hline$k_{p p 2 c}$ & 0.008 \\
\hline$k_{C d c 25 o n}$ & 1.75 \\
\hline$k_{C d c 25 o f f}$ & 0.2 \\
\hline$k_{w e e 1 o n}$ & 0.2 \\
\hline$k_{w e e 1 o f f}$ & 1.75 \\
\hline$k_{\text {plxon }}$ & 1.0 \\
\hline$k_{p l x o f f}$ & 0.15 \\
\hline$k_{\text {apcon }}$ & 1.0 \\
\hline$k_{\text {apcoff }}$ & 0.15 \\
\hline $\mathrm{EC} 50_{C d c 25}$ & 40 \\
\hline EC50 $0_{w e e 1}$ & 40 \\
\hline $\mathrm{EC} 50_{p l x}$ & 40 \\
\hline EC50 apc & 40 \\
\hline$n_{c d c 25}$ & 4 \\
\hline$n_{w e e 1}$ & 4 \\
\hline$n_{p l x}$ & 3 \\
\hline$n_{a p c}$ & 3 \\
\hline $\mathrm{Cdc} 25_{t o t}$ & 15 \\
\hline Wee $1_{\text {tot }}$ & 15 \\
\hline $\mathrm{Plx}_{t o t}$ & 50 \\
\hline $\mathrm{APC}_{t o t}$ & 50 \\
\hline $\mathrm{Cdc} 2_{t o t}$ & 230 \\
\hline$\sigma_{1, \ldots, 9}$ & 0.06 \\
\hline$\eta_{1}$ & 0.4 \\
\hline$\eta_{2}$ & 0.4 \\
\hline
\end{tabular}

Table S2: Parameters in the Ferrell model

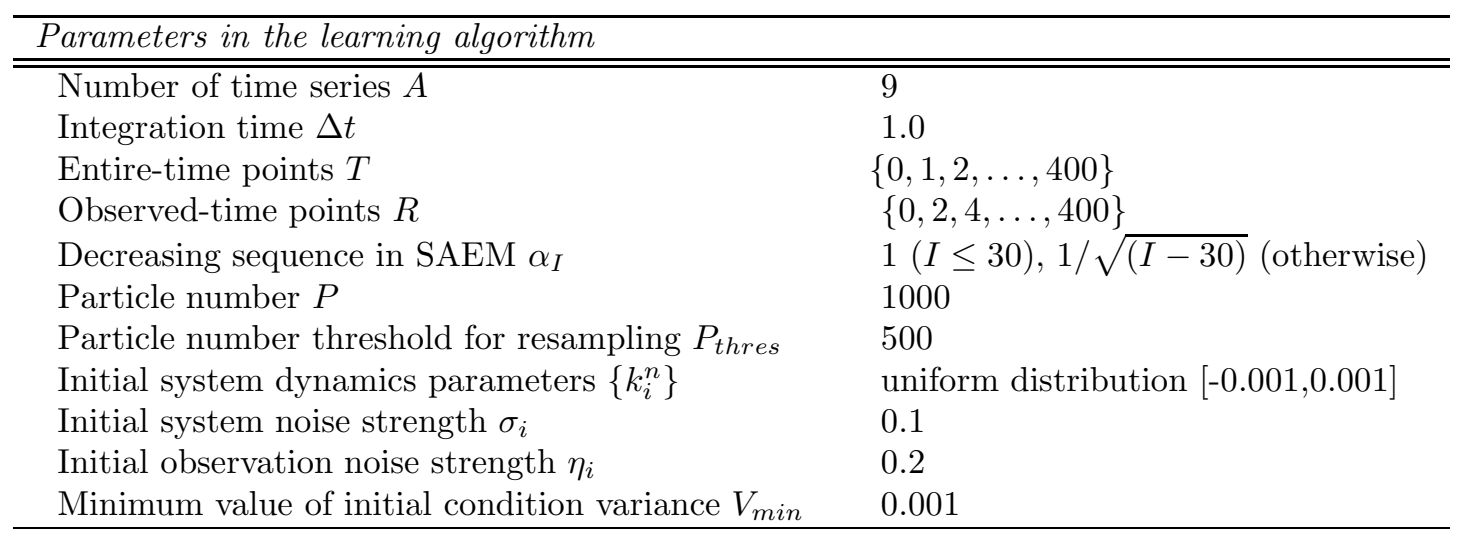

Table S3: Pre-determined parameters used in the learning algorithm. 


\begin{tabular}{ccc}
\hline Parameter & Learned (Tyson model) & Learned (Ferrell model) \\
\hline \hline$k_{1}^{1}$ & 0.00064 & -0.00032 \\
$k_{1}^{2}$ & -0.334 & -0.343 \\
$k_{1}^{3}$ & 0.0655 & 0.0657 \\
$k_{1}^{4}$ & -0.8 & 1.05 \\
$k_{1}^{5}$ & 2.39 & -1.25 \\
$k_{1}^{6}$ & -0.639 & 0.107 \\
$k_{1}^{7}$ & -25.3 & -47.3 \\
$k_{1}^{8}$ & 31.1 & 47.8 \\
$k_{1}^{9}$ & -13.1 & -3.97 \\
$k_{1}^{10}$ & 2.22 & -0.157 \\
\hline$k_{2}^{2}$ & -0.4 & -0.57 \\
$k_{2}^{3}$ & 0.0612 & 0.0637 \\
$k_{2}^{4}$ & -4.62 & -0.9 \\
$k_{2}^{5}$ & 5.47 & 2.41 \\
$k_{2}^{6}$ & -0.805 & 0.000818 \\
$k_{2}^{7}$ & -9 & 61.4 \\
$k_{2}^{8}$ & 22.7 & -61.3 \\
$k_{2}^{9}$ & -15.3 & 18.3 \\
$k_{2}^{9}$ & 1.92 & -2.73 \\
\hline$\sigma_{1}$ & 0.00646 & 0.00199 \\
$\sigma_{2}$ & 0.00703 & 0.00205 \\
\hline$\eta_{1}$ & 0.00334 & 0.00101 \\
$\eta_{2}$ & 0.00621 & 0.00133 \\
\hline
\end{tabular}

Table S4: Learned parameters of the third-order polynomial systems for the Tyson and Ferrell models.

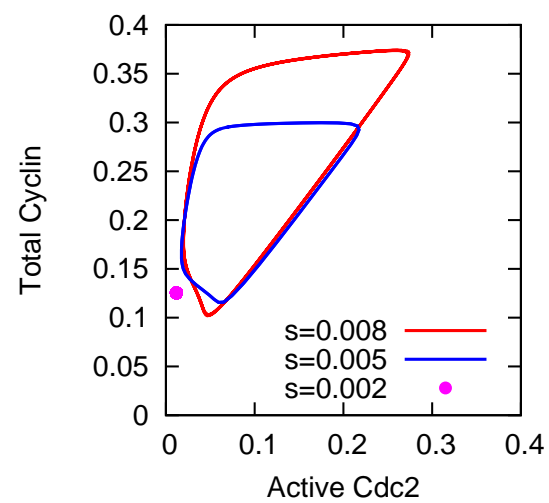

(a)

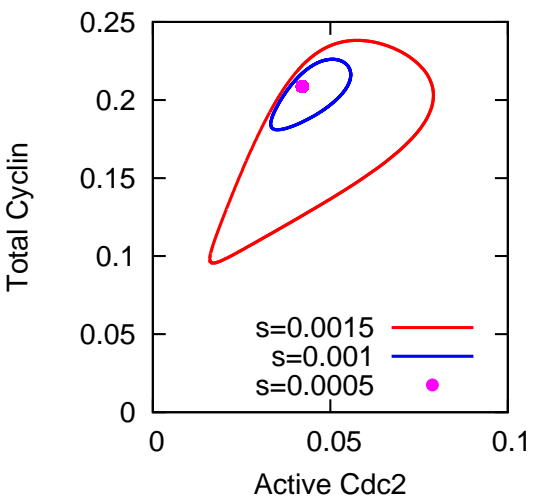

(b)

Figure S1: Time series generated from the (a) Tyson and (b) Ferrell models through the artificial measurement process in the case that both system and observation noises are zero. 


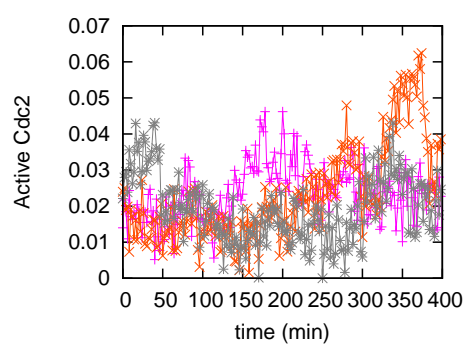

(a)

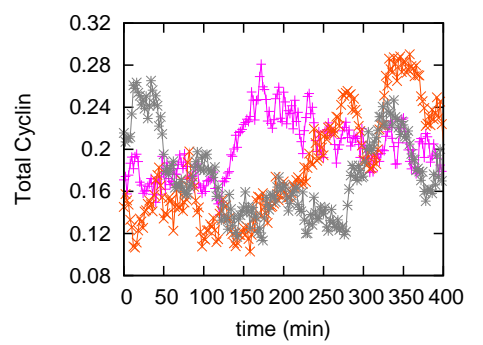

(d)

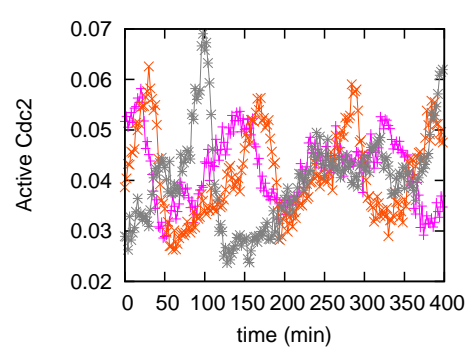

(g)

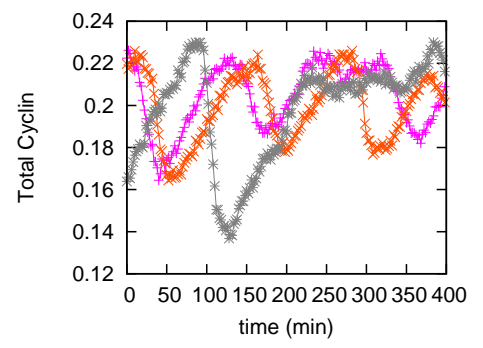

(j)

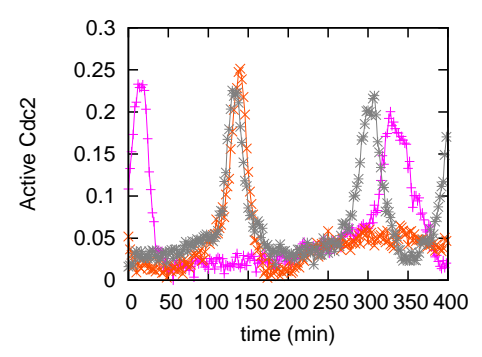

(b)

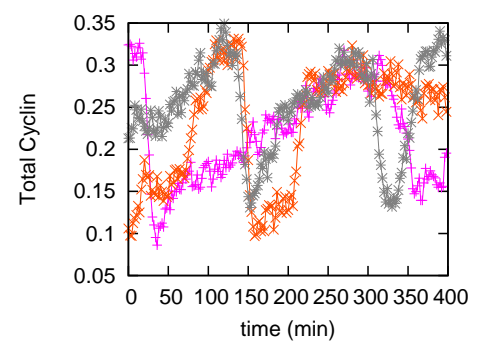

(e)

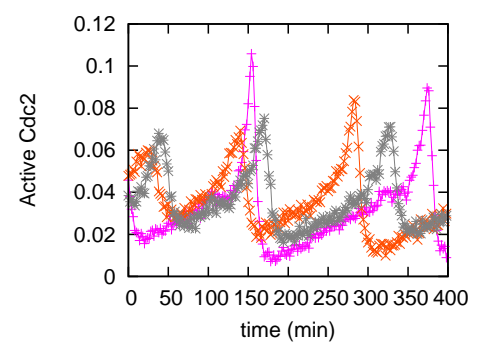

(h)

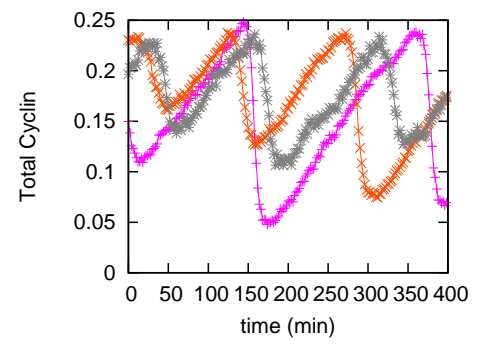

(k)

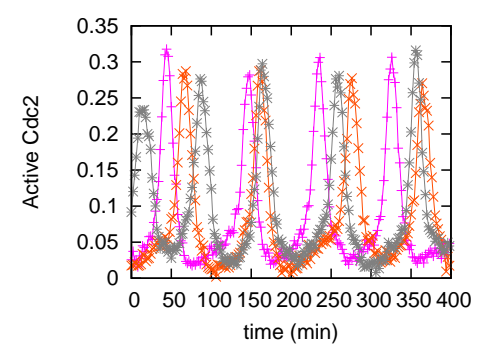

(c)

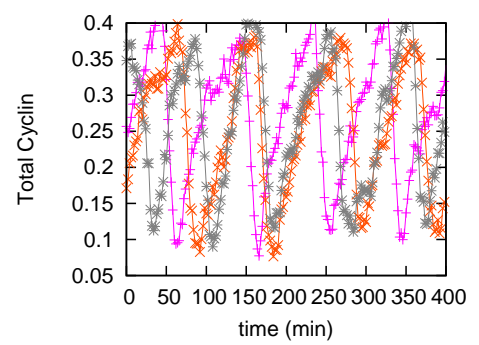

(f)

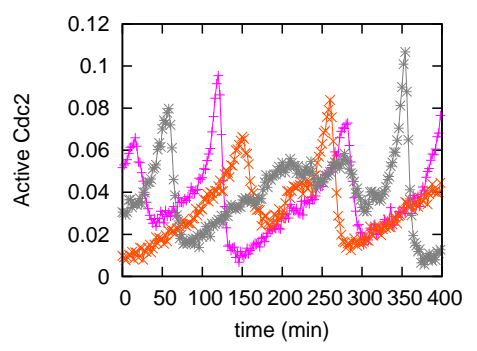

(i)

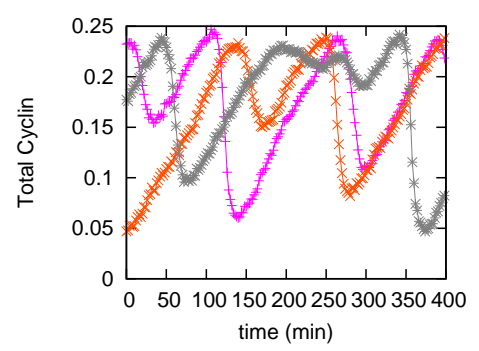

(1)

Figure S2: Noisy time series generated from the (a-f) Tyson and (g-l) Ferrell models through the artificial measurement process. The colors indicate results from independent trials. The values of the bifurcation parameter are $(\mathrm{a}, \mathrm{d}) s=0.002$, (b,e) $0.005,(\mathrm{c}, \mathrm{f}) 0.008,(\mathrm{~g}, \mathrm{j})$ 0.005, $(\mathrm{h}, \mathrm{k})$ 0.001, and $(\mathrm{i}, \mathrm{l})$ 0.0015, respectively. 


\section{References}

[1] P. E. Kloeden and E. Platen, Numerical Solution of Stochastic Differential Equations (Stochastic Modelling and Applied Probability), Springer, 3rd edition, 1999.

[2] A. P. Dempster, N. M Laird, and D. B Rubin, Maximum likelihood from incomplete data via the EM algorithm, Journal of the Royal Statistical Socety, Series B, 39:1 - 38, 1977.

[3] B. Delyon, M. Lavielle, and E. Moulines, Convergence of a stochastic approximation version of the EM algorithm, The Annals of Statistics, 27:94-128, 1999.

[4] N.J. Gordon, D.J. Salmond, and A.F.M. Smith, Novel approach to nonlinear/nonGaussian Bayesian state estimation, Radar and Signal Processing, IEE Proceedings F, 140:107-113, 1993.

[5] G. Kitagawa, Monte Carlo Filter and Smoother for Non-Gaussian Nonlinear State Space Models, Journal of Computational and Graphical Statistics, 5:1-25, 1996.

[6] M.S. Arulampalam, S. Maskell, N. Gordon, and T. Clapp, A tutorial on particle filters for online nonlinear/non-Gaussian Bayesian tracking, IEEE Transactions on Signal Processing, 50:174-188, 2002.

[7] R. L. Honeycutt, Stochastic runge-kutta algorithms. I. White noise, Physical Review A, 45:600-603, 1992.

[8] B. Novak and J. J. Tyson, Numerical analysis of a comprehensive model of M-phase control in Xenopus oocyte extracts and intact embryos, Journal of cell science, 106:1153-68, 1993.

[9] T. Y.-C. Tsai, Y. S. Choi, W. Ma, J. R. Pomerening, C. Tang, and J. E. Ferrell Jr., Robust, tunable biological oscillations from interlinked positive and negative feedback loops, Science, 321:126-9, 2008.

[10] J. R. Pomerening, S. Y. Kim, and J. E. Ferrell Jr., Systems-level dissection of the cell-cycle oscillator: bypassing positive feedback produces damped oscillations, Cell, 122:565-78, 2005.

[11] B. Novak and J. J. Tyson, Modeling the Cell Division Cycle: M-Phase Trigger, Oscillations, and Size Control, Journal of Theoretical Biology, 165:101-134, 1993. 